Ortega Centella, Visitación.

Doctoranda en Arte. Universidad de Granada, Departamento de Pintura.

\title{
Un proceso análogo entre la fenomenología de la oscuridad y la destrucción de la memoria.
}

\author{
TIPO DE TRABAJO
}

Comunicación.

PALABRAS CLAVE

Oscuridad, Destrucción, Arte contemporáneo.

KEY WORDS

Darkness, Destruction, Contemporary art.

RESUMEN

Situando como sedimento inicial el concepto de oscuridad, así como aquellos que derivan de su terminología, como puede ser el oscurantismo, o de su relación fisiológica con el medio, como el color negro, nos aproximamos en el presente artículo a las relaciones posibles entre los procesos de destrucción y deconstrucción, en términos derridianos, asociados a la memoria. Es decir, como procesos análogos al concepto oscuridad, se presenta, a través de artistas contemporáneos, como Christian Boltanski (1944- ) o Michael Landy (1963- ), entre otros, las consecuencias más inmediatas que trascienden al espectador desde la concepción de la oscuridad, haciendo alusión al pensamiento de Jacques Derridá (1997), que tienen cabida en la memoria del sujeto. Se pretende aquí presentar un segmento del estudio que llevo a cabo en mi tesis doctoral. Con la premisa de una simbología negativista del concepto de oscuridad, así como aquellos otros derivados etimológicamente del mismo, nos aproximamos a los procesos, de carácter existencialista, que se perciben en las obras presentadas, para configurar y visualizar aquellos otros que apelan a lo oculto.

A modo de conclusión, se presenta una síntesis reflexiva sobre aquellos conceptos tratados en nuestro artículo, que a su vez se identifican con los procesos de destrucción y deconstrucción análogos a la fenomenología de la oscuridad.

\section{ABSTRACT}

Placing such as initial sediment the concept of the darkness, as well as those that derive from their terminology, such as obscurantism, or with the physiological relationship with the place, as the black color, in this article we approach to the possible relationships between the processes of destruction and deconstruction, in derridean terms, associated with the memory. That is, as similar processes to the concept darkness, are presented through by contemporary artists, such as Christian Boltanski (1944) and Michael Landy (1963), among others, the most immediate consequences that transcend the viewer from the conception of the dark, alluding to the thought of Jacques Derrida (1997), which take place in the memory of the subject. It is intended here present a segment of the study that I am doing in my doctoral thesis. With the premise of a naysayer symbology of the concept of darkness, as well as those derived etymologically the same, we approach the processes, of existentialist character, perceived in the works presented, to configure and display those that appeal to the occult.

To conclude, a thoughtful synthesis of those concepts discussed in our article is submitted, which in turn are identified with the processes of destruction and deconstruction analogous to the phenomenology of darkness. 


\section{CONTENIDO}

\section{INTRODUCCIÓN}

Plantear a través de la analogía, un discurso donde confluyan tanto el poder de la destrucción, la metodología deconstructivista, la fenomenología de la oscuridad y la memoria, no es de fácil cometido, teniendo en cuenta los numerosos estudios de carácter existencialistas que se han desarrollado sobre los mismos a lo largo de la historia. Pero es, a través de una mirada patológica, el miedo a la oscuridad, la acluofobia ${ }^{1}$, desde donde se plantea las posibles relaciones fenomenológicas que confluyen entre sí, desde el constructo oscuridad, de donde se parte en la investigación que desarrollo en los estudios de doctorado en los que me encuentro inmersa.

\section{LA OSCURIDAD COMO DESTRUCCIÓN}

La acción de destruir cualquier cosa, material o espiritual, o causar un daño o ruina muy grande, significa que se está utilizando la llamada destrucción. El acto de destruir y descomponer algo con cualquier objetivo, es decir, ya sea por el mero hecho de que se produzca su desaparición, total o parcial, o se pretenda reestablecer a posteriori, marca el inicio que conlleva un nuevo planteamiento sobre lo que se aplica. Haciendo alusión al pensamiento heideggeriano, la destrucción no alude a la aniquilación total, sino que se asiste a un proceso de desmontaje o desmantelamiento. Un planteamiento que anticipa al término deconstrucción acuñado por Jacques Derrida para identificar la división de carácter infinita.

Una definición sobre el término realizada por Ortiz-Osés y Lanceros (1998) en su edición digital sobre Derrida:

Deconstruir consiste, en efecto, en deshacer, en desmontar algo que se ha edificado, [...] pero no con vistas a destruirlo, sino a fin de comprobar cómo está hecho ese algo, cómo se ensamblan y articulan sus piezas, cuáles son los extractos ocultos que lo constituyen. ${ }^{2}$

Roberto Ferro (1992) también analizará el concepto derridiano de la deconstrucción a través del lenguaje escrito de la siguiente manera:

La desconstrucción emerge en la deriva de un pensamiento que tiene como hilo conductor a la escritura, y se despliega como una escritura de la escritura, que implica e insiste en "otra" lectura, no sometida a un campo de legibilidad dominado por la impronta hermenéutica del sentido del querer-decir de un discurso; una lectura que revele su fondo de ilegibilidad, es decir, las instancias no intencionales inscritas en los sistemas significantes de un discurso que lo configuran como texto, una lectura que trastorne la posibilidad de ser compactada como expresión de un sentido, o que deliberadamente se presente como efecto sometiéndose a la legalidad forzada de la doble ilusión metafísica: la de la consciencia constitutiva del sentido y la ilusión de la plenitud de la presencia del referente. ${ }^{3}$

El concepto de deconstrucción aplicado al fenómeno de la oscuridad acontece en la misma medida que se aplica a la escritura, es decir, se establece una doble lectura de un mismo concepto que se emplea sobre cualquier circunstancia, hecho u objeto. La oscuridad formaría parte de aquella reflexión que queda subordinada y oculta bajo el discurso principal que es iluminado, a nivel metafórico, con un foco de luz, aportando el conocimiento y la comunicación. Una luz metafórica que a su vez alude a todo aquello que es percibido por la vista, o cualquier otro sentido, y una oscuridad que refleja lo desconocido, aquello que resulta difícil identificar. La oscuridad formaría parte del plano sometido y dominado por el primer impulso del querer-mostrar en un discurso determinado; que no muestra la esencia, sino que se oculta bajo el despliegue de los distintos significantes extraídos de un mismo concepto. Es esta metodología deconstructiva de la que se nutre la oscuridad, que utiliza la destrucción para despiezar un discurso y posicionar el plano que más interesa a la estructura de poder dominante. Heidegger, en este sentido, sitúa a Kant $(1927)^{4}$ como el primero y el único que se dejó empujar en la fuerza de la dimensión del fenómeno de la temporariedad, aventurándose al dominio oscuro, que en relación al planteamiento, se expone la cita extraída de El ser y el tiempo (1927):

${ }^{1}$ Acluofobia (del gr. Achlúos, niebla, y phóbos, terror) es la fobia o miedo desmedido a la oscuridad, y es la premisa patológica que se desarrolla en la investigación. Un miedo irracional que se caracteriza por el pánico provocado en situaciones donde la no-luz está presente haciendo muy complejo la percepción visual de los elementos que componen el espacio así como lo limítrofe entre el sujeto, lo corpóreo y el espacio, el lugar. Habitar lugares oscuros o simplemente imaginar situaciones donde la luz no está presente, hace que el sujeto se encuentre en situación de riesgo, realizando cambios fisiológicos y físicos corporales para afrontar el peligro real o imaginario que está por ocurrir.

${ }^{2}$ PERETI, Cristina. Deconstrucción. Entrada del Diccionario de Hermenéutica por A. Ortiz-Osés y P. Lanzeros (directores). Bilbao: Universidad de Deusto, 1998. Edición digital de Derridá en Castellano. [En línea] Consultado [07-01-2010] Disponible en: www.jacquesderrida.com.ar/comentarios/perretti 2.htm

${ }^{3}$ FERRO, Roberto. Escritura y desconstrucción: lectura (h)errada con Jacques Derrida. Buenos Aires: Editorial Biblos, 1995, p.117.

4 "Lo esencial, en la magia negra de los oscurantistas [...] tiende a ennegrecer la imagen del mundo y a oscurecer nuestra idea de la existencia [...] ¿Es posible utilizar para este fin a Kant mismo? Diré más: ¿es posible que, según su propia declaración tristemente famosa, haya querido él mismo algo semejante, al menos de una manera pasajera: abrir una ruta a la fe, asignando límites a la ciencia?" FRIEDRICH, Nietzsche. El viajero y su sombra. VERGARA, Carlos (trad.). Madrid: Ediciones EDAF, 1999, p. 27. 
Este esquematismo de nuestro entendimiento en lo que respecta a los fenómenos y a su mera forma, es un arte escondido en las profundidades del alma humana, cuyos verdaderos artificios difícilmente arrancaremos nunca a la naturaleza, ni pondremos al descubierto ante nuestros ojos. ${ }^{5}$

Con este planteamiento se sitúa a la oscuridad en la parte más profunda del ser, que no puede nunca llegar a ser conocida en su totalidad. Por lo tanto, el dominio de la oscuridad, queda en un plano inalcanzable ante la vista y al entendimiento verdadero ya que forma parte del fondo del ser. Esta visión negativista del concepto confirma la verdadera connotación de carácter oculto que se ha otorgado al fenómeno a lo largo de la historia. Un concepto que se encuentra en el plano invisible e inaccesible a la percepción humana, pero que a su vez transciende de tal forma, que altera el curso de los demás componentes que la conforman.

A modo de ejemplo, se destaca una de las acciones que se han llevado a cabo a lo largo de la historia con la principal intención de acabar con la historicidad y el pensamiento desarrollado a través de la misma, como la acción de la quema de libros. Este plan, que destruye todo aquello que apela al desarrollo del conocimiento, aquellos que los poderes gobernantes desestiman por su contenido y por la defensa de unos ideales determinados y no apoyan su difusión, no comparte el principio que alude al concepto de durée de H. Bergson: "implica por un lado, la contemporaneidad o coexistencia del pasado y del presente, para lo cual es indispensable una memoria ontológica, y por otro, diversos grados de fusión entre el pasado y el presente en dirección al porvenir" ${ }^{6}$. Es decir, si deben de coexistir el pasado y el presente para que tenga lugar la memoria ontológica según la tesis bergsoniana, aquí no se cumpliría como tal, pero si se produciría esa coexistencia entre ambos significados, siendo el otorgado en el pasado el que aporta la negatividad al constructo del presente. El pasado formaría parte de la sombra, de lo oculto y lo confuso que complementaría al presente. La memoria, por lo tanto, quedaría supeditada a los discursos oscurantistas devenidos de un pasado tenebroso, quizás, sin testigo referente alguno sobre su existencia. Aplicar el concepto bergsoniano a la fenomenología de la oscuridad conlleva la aceptación de que su significación primigenia, utilizada para determinar uno de los periodos más antiguos de la civilización occidental de la cultura griega, como la Edad Oscura, ha sido peyorativa. Su uso, que establece aspectos negativos relacionados con la pérdida, la destrucción y la muerte, han concluido que ese pasado quede en nuestro presente, avivando hasta nuestros días las acepciones etimológicas configuradas a lo largo de la historia.

Como apunta Fernando Vidal: "la vida ha de entenderse mediante la duración" memoria. Por lo tanto, la destrucción que supone la desaparición de la durabilidad en el tiempo, hace que no convivan los acontecimientos pasados con los presentes, suponiendo así la desaparición de una posible memoria ontológica. Si la oscuridad representa esa falta de fuentes sobre el pasado, que a su vez no coexiste con el presente, provoca entonces la imposibilidad de plantear la historicidad acontecida. Esto supone un desconocimiento a través de un planteamiento destructivo que no contempla el porvenir, una oscuridad que apela a la destrucción como arma letal de la memoria ontológica perteneciente a una cultura. Esta oscuridad, planteada desde el discurso destructivo, sitúa a la muerte, entendida como el cese de cualquiera de los elementos que conforman la vida, como estrategia principal. La aniquilación y la destrucción, sinónimos de muerte, representan la oscuridad a la que se hace referencia en este artículo. Una oscuridad capaz de encubrir aquellos sucesos que conlleva a la desaparición de los elementos que conforman una cultura y que son representativos en la sociedad.

La muerte se contempla a través de las distintas analogías que alude el concepto de destrucción y la desaparición de la memoria. La muerte de la memoria como consecuencia de la destrucción del conocimiento creado a lo largo de la historia, la aniquilación de los saberes de una sociedad determinada por pertenecer a la parte subyugada de los poderes gobernantes. Una muerte que:

Señala el momento a partir del cual dejamos de hablar del interfecto en presente o futuro [...] y empezamos a hablar de él en pasado. [...] toda política de la muerte es siempre, simultánea e indefectiblemente, una política de la memoria. ${ }^{8}$

Según Néstor A. Braunstein "la memoria es previa. Es fundadora del ser [...] La memoria no sería un archivo de documentos sino una construcción enriquecida por la imaginación" ${ }^{9}$. Desde la ciencia cognitiva, la memoria se define como aquella que "interviene en todos los procesos mentales: pensamos, hacemos juicios críticos, resolvemos problemas y damos opiniones [...] un complicado sistema dinámico de almacén único y diferente en cada individuo" ${ }^{10}$. De acuerdo con esta definición, el papel que ocupa la memoria en el desarrollo de la cultura y del conocimiento es fundamental para el proceso evolutivo. Si a través de la memoria se edifican hechos

\footnotetext{
${ }^{5}$ Cita extraída de Kritik der reinen Vernunft, pp. 180 s. en HEIDEGGER, Matin. El ser y el tiempo. Madrid: Fondo de Cultura Económica de España, 1998, p. 34.

${ }^{6}$ BERGSON, Henri. Duración y simultaneidad: (a propósito de la teoría de Einstein). MARTIN, Jorge (traductor). Buenos Aires: Ediciones del Signo, 2004, p. 22.

${ }^{7}$ VIDAL, Fernando. Piaget antes de ser Piaget. Madrid: Ediciones Morata, 1998, p. 68.

${ }^{8}$ CASQUETE, Jesús [et al.] Políticas de la muerte. Usos y abusos del ritual fúnebre en la Europa del siglo XX. Madrid: Ediciones Catarata, 2009, p. 342.

${ }^{9}$ BRAUNSTEIN, Néstor A. Memoria y espanto o el recuerdo de la infancia. México: Siglo XXI Editores, 2008, p. 10.

${ }^{10}$ VARELA R., Margarita; ÁVILA A., M. R., y IMELDA F., Teresa. La memoria. Definición, función y juego para la enseñanza de la medicina. Madrid: Editorial Médica Panamericana, 2005, p.19.
} 
acontecidos, y ésta, ha sido destruida por los diferentes organismos de poder de las civilizaciones desplegadas, significa que ha quedado subordinada bajo las reflexiones oscurantistas que se han desarrollado para tal fin. Sobre la memoria, Ángel Nogueira (2001) apunta que:

La memoria no es sólo el producto almacenado de lo que experimentamos, sentimos e imaginamos; la memoria es, sobre todo, un poderoso sistema de adquisición y transmisión de conocimientos que nos permite revivir el pasado, interpretar el presente y planificar el futuro. Sin la memoria no existiría vínculo posible entre el pasado y el futuro. ${ }^{11}$

La destrucción de la memoria, por lo tanto, significaría la aniquilación del vínculo existente entre el pasado y el presente, y la incapacidad de planificar el futuro con reminiscencias prevalecientes. No sólo se alude a la memoria individual, que a su vez se nutre de agentes externos al individuo, sino que apelamos a la memoria colectiva como arma constructiva y permanente sobre los hechos acontecidos y los posibles venideros. Y al referirnos a la memoria colectiva, también se pone de manifiesto todos aquellos elementos que la constituyen y permiten su permanencia en el tiempo, como son los libros y escritos, fundamentalmente creados para la difusión, transmisión y desarrollo del conocimiento en una dimensión cronológica, espacial y temporal.

CHRISTIAN BOLTANSKI, MICHAEL LANDY Y RAQUEL WITHREAD.

Como obra cargada de simbolismos de carácter existencialista, relacionados con el paso del tiempo, la soledad, el vacío, y por ende, la muerte, que además, es conformada en un espacio-temporal estableciendo un vínculo ritualístico con el lugar donde se exhibe, se presenta la obra Tierra de nadie, 2010 de Christian Boltanski.

Su obra muestra el reflejo del pensamiento occidental respecto a la muerte, al luto y la desgracia. Tierra de nadie, 2010 (re)presenta una alegoría a la oscuridad mediante la ausencia-presencia, la desaparición y el vacío; y la memoria participa en la configuración del espacio y de los objetos, en la medida que éstos ocupan el lugar. Se trata de una obra monumental creada para el segundo proyecto anual del Park Avenue Armory's de Nueva York, con unos 5000 metros cuadrados de extensión del Wade Thompson Drill Hall, donde se ocupa todo el espacio con miles de piezas de ropa usada y con un acompañamiento sonoro que retumba en toda la sala de los fuertes latidos del corazón. Es uno de los proyectos más monumentales que Boltanski ha creado para los Estados Unidos, cuyas alusiones poéticas explora los conceptos más profundos de la identidad humana, la memoria y la muerte.

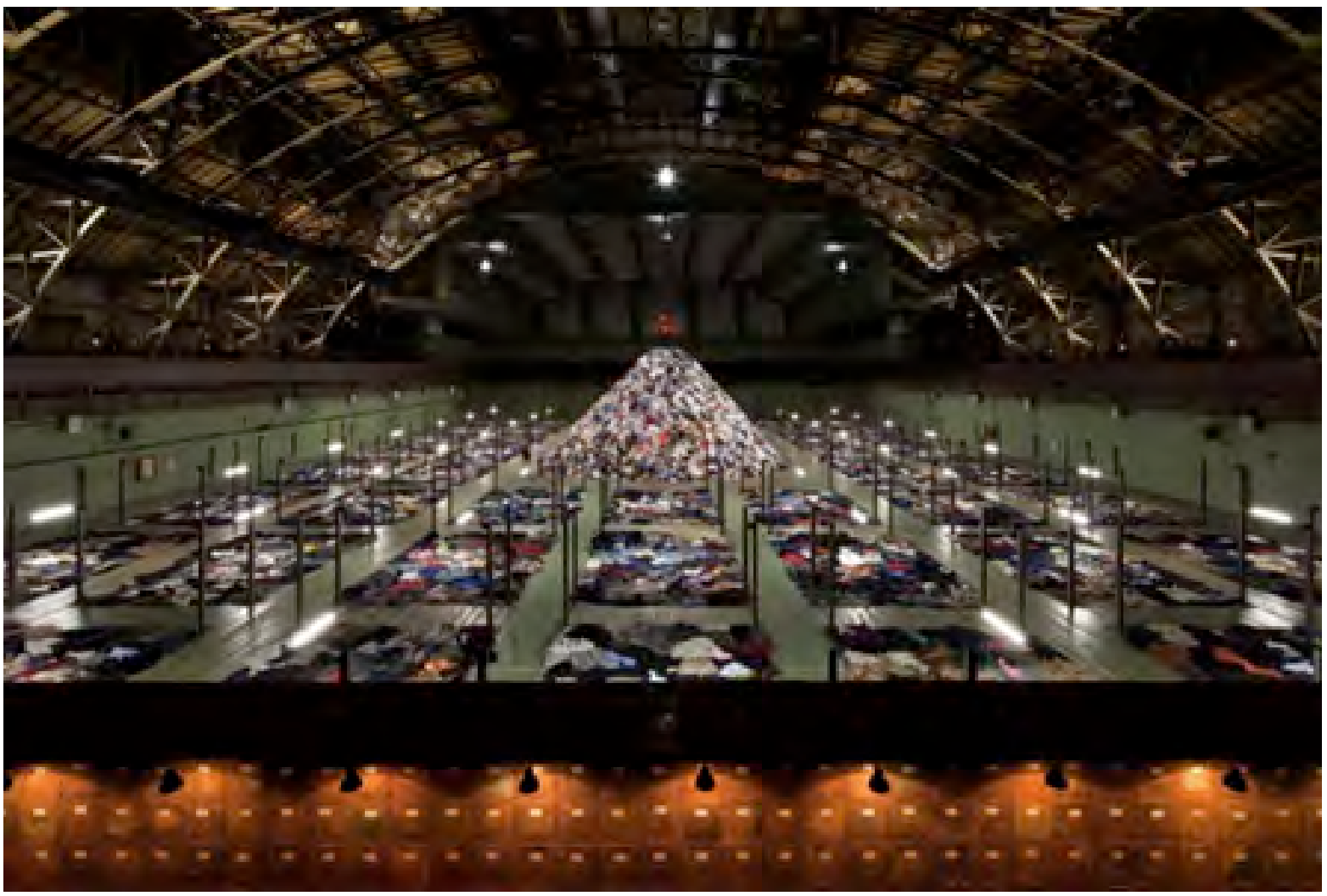

Ilustración 1. Christian Boltanski. Tierra de nadie, 2010. Vista general de la instalación creada para el segundo proyecto anual del Park Avenue Armory's de Nueva York. Fotografía de: Christian Boltanski

\footnotetext{
${ }^{11}$ NOGUEIRA D., Ángel. "Huellas del conocimiento". Psicología cognitiva de la memoria. Revista Anthropos. 2001, n¹89/190, p.3.
} 
El espectador que se adentra en la instalación, en primer lugar se enfrenta a una gran pared de unos 20 metros de longitud, compuesta por unas $\mathbf{3 0 0 0}$ latas de galletas (Ilustración 1), todas ellas oxidadas, y que impiden la visión de toda la superficie restante. Tras el gran muro, el espectador se encuentra con un paisaje de carácter fantasmal compuesto por 45 parcelas rectangulares determinadas por montañas de ropas recicladas, destacando entre ellas una montaña de unas 30 toneladas de ropa. Es en esta última donde se sitúa una grúa (ilustración 2), que alude a la ceguera, que maniobra con una mano mecánica que alza la ropa al azar, para posteriormente, dejarla caer nuevamente en el montículo. Una acción que invita a la reflexión sobre el azar y el destino de toda experiencia humana. A estos sonidos mecanizados se añaden los sonidos de los fuertes latidos del corazón humano, que se emiten en toda la sala. Los espacios, cuestionados mediante la situación de los objetos, son intervenidos y renombrados como nuevos lugares simbólicos, quedando la experiencia trasferida a través de la memoria colectiva compartida.

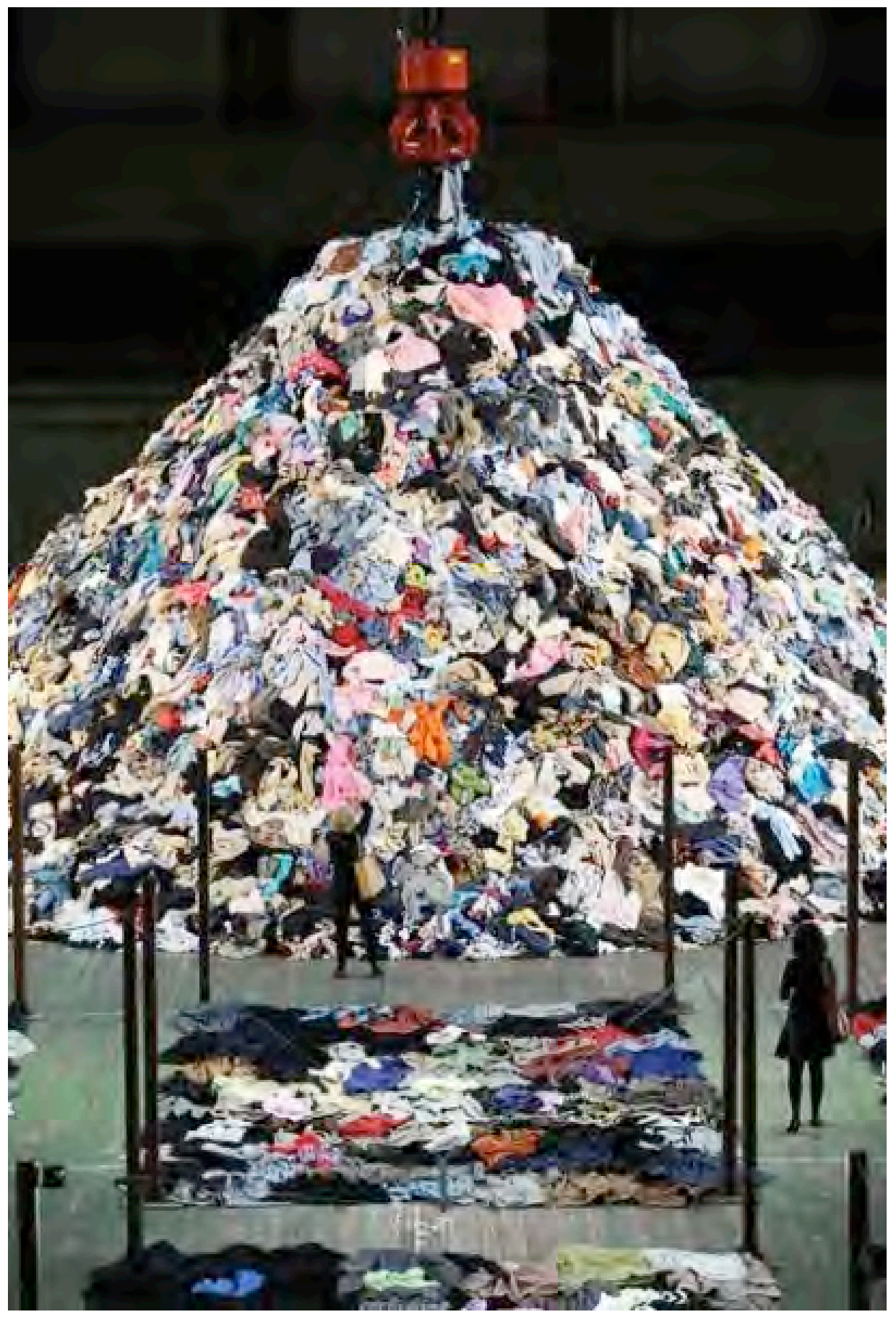

Ilustración 2. Christian Boltanski. Tierra de nadie, 2010. Detalle del montículo principal de 30 toneladas donde se encuentra el brazo de la grúa mecanizada. Park Avenue Armory's de Nueva York. Fotografía de: Christian Boltanski 
De la misma forma, en la obra titulada Judenplatz Holocaust Memorial, 2000 (ilustración 3) de Raquel Whiteread, localizada en la 1010 Judenplatz de Viena, invita a reflexionar sobre el lugar de la memoria colectiva que se comparte como espacio fusionado y que esboza las distintas memorias sin recuerdos de los individuos. Consiste en una pieza realizada en hormigón, cuyas paredes están formadas por hileras de libros, como si de estantería de una biblioteca se tratase, colocados hacia el exterior, es decir, con las páginas hacia fuera, conmemorando la expulsión de los judíos, y aludiendo además a la acción de la quema de libros del pueblo judío realizada por los nacionalsocialistas en 1933. Se encuentra situada en las ruinas de la antigua sinagoga, y conmemora los 65000 judíos austriacos asesinados por los nazis. Una biblioteca de hormigón, en la que no se puede acceder, donde los libros nadie los puede leer, y donde las oraciones quedan ocultas e inaccesibles.

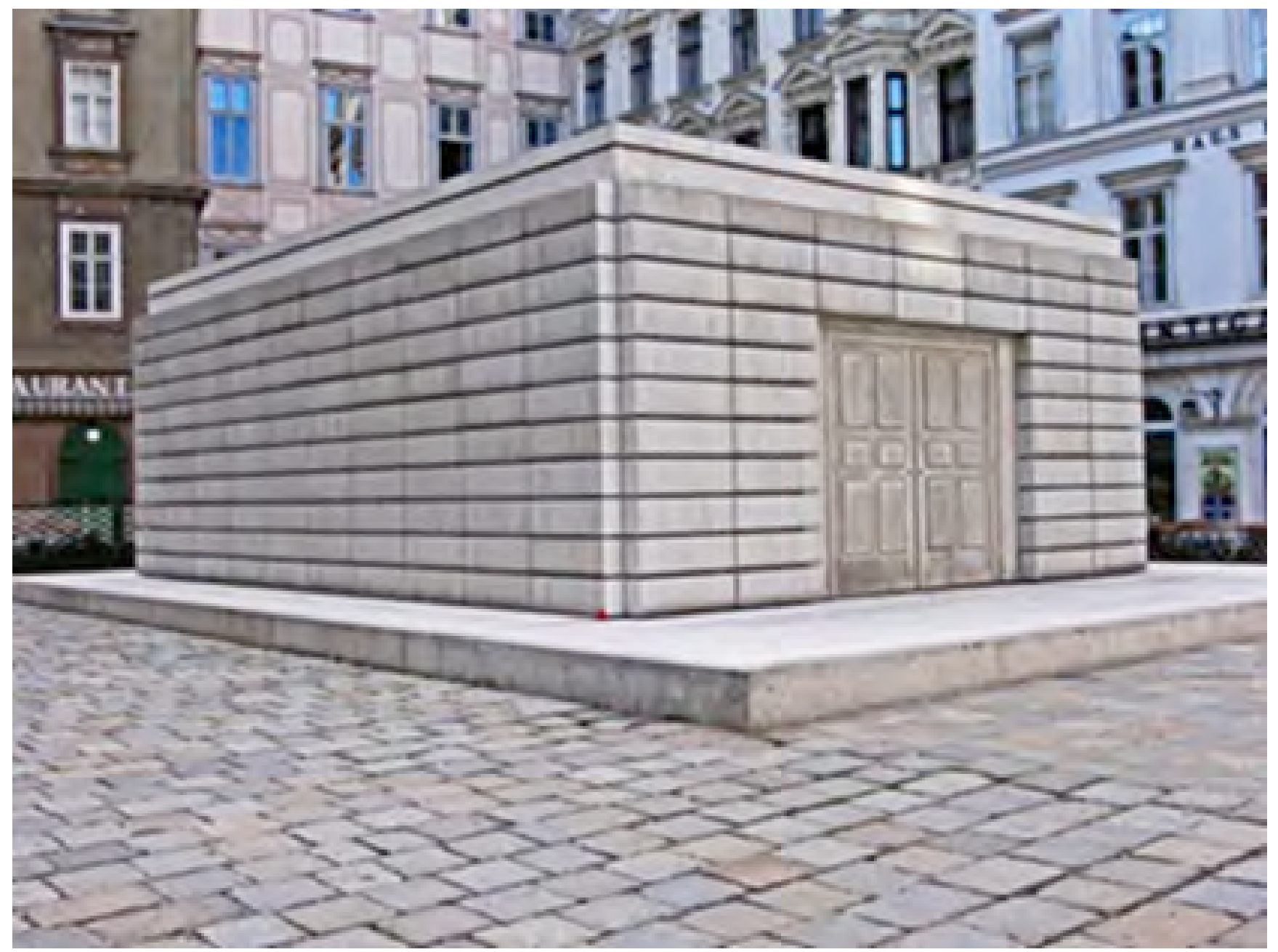

\section{Ilustración 3. Rachel Whiteread. Judenplatz Holocaust Memorial, 2000. Obra situada en la Judenplatz de Viena, Austria. Fotografía de: Rachel Whiteread}

Su monumentalidad también hace eco de los búnkeres militares de la Segunda Guerra Mundial, así como de los acontecimientos que narran las historias de un tiempo determinado del pasado, concretamente de las acciones llevadas a cabo parra la exterminación del pueblo judío. Se pone de manifiesto en esta obra la utilización de toda una memoria histórica-cultural de un pueblo que ha sido perseguido a lo largo de la historia por los diferentes organismos de poder. La alegoría a la transmisión del conocimiento a través de los libros, y como su destrucción, también forma parte de la destrucción propia de la una cultura, dejando en evidencia la capacidad de destrucción que puede desarrollar el ser humano. La oscuridad queda latente en el proceso de aniquilación de la especie que no comparte una misma visión ontológica, o cuyas costumbres distan en cierta medida de la pertenecientes a los intereses gobernantes. Aquí, se mantiene el principio de destrucción, como la muerte final del discurso, del objeto, como analogía a la destrucción de toda una cultura. El fin último de esta destrucción, sitúa a la oscuridad en lo más profundo del ser, cuya percepción es mucho más compleja y difícil de descifrar.

En la medida que se aplica el concepto de destrucción en la obra Break Down, 2001 (figura 4) de Michael Landy (1963- ), que significa romper, descomponer o desglosar, se presenta a continuación como ejemplo que utiliza la metodología deconstructiva en la creación (ilustración 4). 


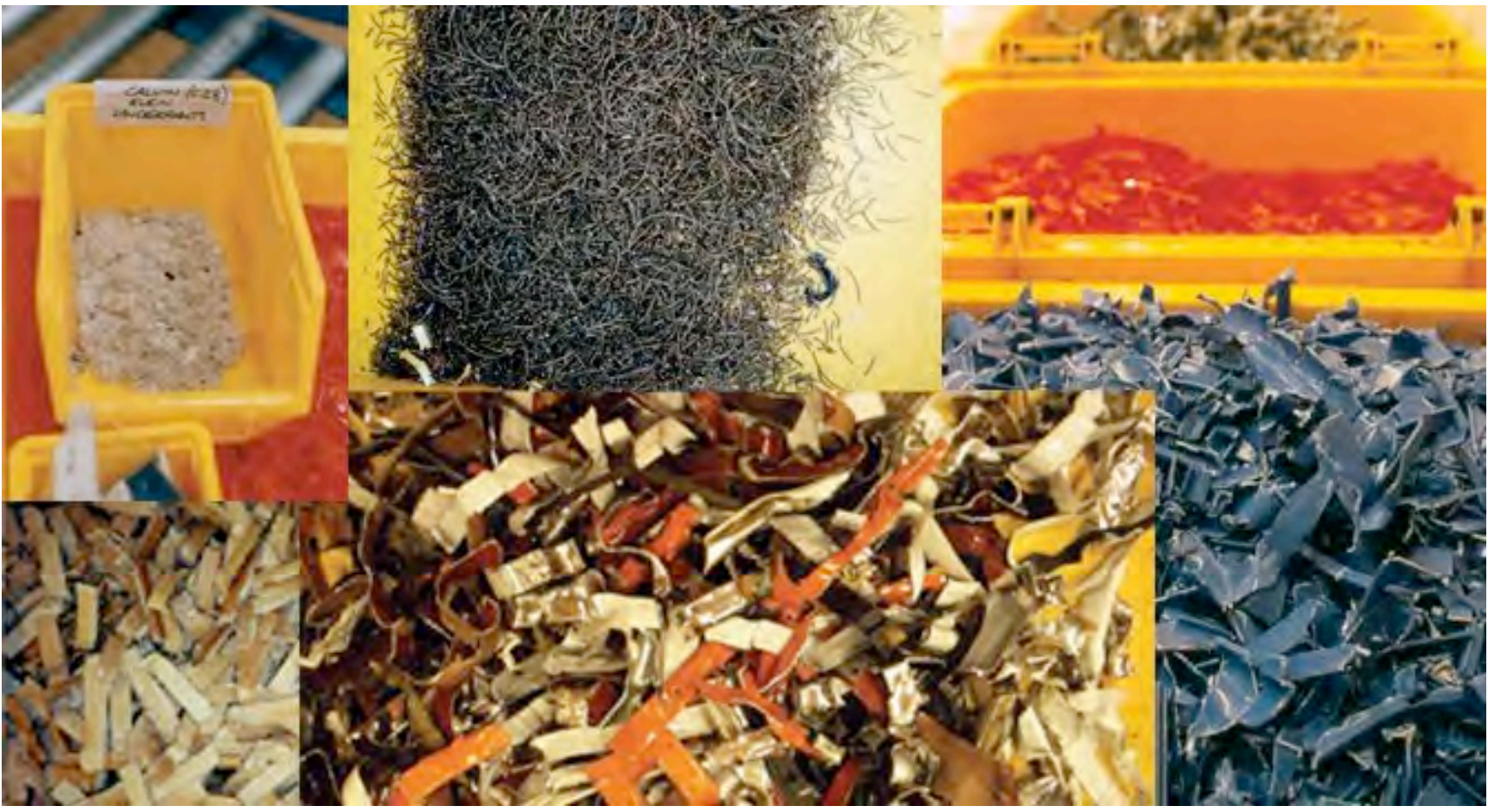

\section{llustración 4. Michael Landy. Sección de la categorización de las piezas destruidas de la obra Break up, 2001 en un antigua C\&A en Oxford Street, Londres. Fotografía: Michael Landy}

La performance de Landy consistía en destruir sistemáticamente todas sus pertenencias acumuladas a lo largo de su vida: fotografías familiares u objetos personales, incluido su coche. El objetivo principal de la obra se rige sobre la categorización de las diferentes piezas que componen un todo, destrozadas, acumuladas en una base de datos donde finalmente son etiquetadas $y$ empaquetadas. Durante tres años, junto a un equipo de ayudantes vestidos con atuendos azules, como si de empelados de una fábrica se tratara, tuvo lugar la realización de la obra que reducía el objeto a la cantidad de materiales que lo componían, para, a continuación, ser aplastados y granuladas a su mínima expresión, quedando así, irreconocibles. La obra se desarrolló, paradójicamente, en una antigua fábrica que mantenía una línea de producción en perfectas condiciones de uso. La analogía semejantes respecto a nuestro tema de estudio que conforma el fenómeno de la oscuridad, se presentan en esta obra a través de la metodología procesual "deconstructiva" por la cual se conocen los objetos ocultos y desconocidos que conforman una misma pieza. Más allá de esta concepción espacial del objeto, la reflexión más profunda que alcanza la pieza, quizás podemos denominarla oscuridad, se centra en la cultura consumista oculta bajo las necesidades primarias de supervivencia. Es decir, todo ser necesita de unos elementos formales que conforman su vida cotidiana, pero el consumismo de la modernidad, ha hecho que estos elementos, a su vez superfluos, acaban generando necesidades, aunque gran parte de ellas sean artificiales, productos de los agentes publicitarios de las grandes empresas.

\section{A MODO DE CONCLUSIÓN}

A modo de conclusión, afirmaremos, en primer lugar, la relación existente entre la fenomenología de la oscuridad y los factores relacionados con la destrucción de la memoria ontológica, el proceso deconstructivo y en última instancia la muerte, relacionada esta última, con el vacío, la soledad y el sometimiento, a través de las propuestas artísticas aquí presentadas, donde se cumplían los principios de la teoría bergsoniana de la duración. Apuntaremos, que la muerte de la cultura, conlleva una muerte de la sociedad, donde el conocimiento deja de expandirse para forma parte de la usencia, la memoria y el vacío en la cultura popular, entendiéndose esta última como la define Hall Stuart (1998), como todo aquello que la gente hace o ha hecho en el pasado ${ }^{12}$. Por lo tanto, la destrucción de la memoria simboliza a un mismo tiempo una ocultación, una ausencia que queda de manifiesto por su presencia y por su capacidad de desvelar reminiscencias de un pasado próximo que a su vez se utiliza para (re)construir un presente. Y es ahí donde se encuentra la oscuridad.

\footnotetext{
12 "Popular culture is all the things that the people do or have done", constituyendo la segunda definición que Hall aporta sobre el término de cultura popular en Notes on Deconstructing 'the popular'. En SAMUEL, Raphael (ed.) People's History and Socialist Theory. London: History Workshop Journal, 1981, p. 234.
} 


\section{FUENTES REFERENCIALES}

BERGSON, Henri. Duración y simultaneidad: (a propósito de la teoría de Einstein). MARTIN, Jorge (traductor). Buenos Aires: Ediciones del Signo, 2004.

BRAUNSTEIN, Néstor A. Memoria y espanto o el recuerdo de la infancia. México: Siglo XXI Editores, 2008.

CASQUETE, Jesús [et al.] Políticas de la muerte. Usos y abusos del ritual fúnebre en la Europa del siglo XX. Madrid: Ediciones Catarata, 2009.

FERRO, Roberto. Escritura y desconstrucción: lectura (h)errada con Jacques Derrida. Buenos Aires: Editorial Biblos, 1995.

FRIEDRICH, Nietzsche. El viajero y su sombra. VERGARA, Carlos (trad.). Madrid: Ediciones EDAF, 1999.

HEIDEGGER, Matin. El ser y el tiempo. Madrid: Fondo de Cultura Económica de España, 1998.

NOGUEIRA D., Ángel. “Huellas del conocimiento”. Psicología cognitiva de la memoria. Revista Anthropos, 2001, nํ189/190, p.3.

PERETI, Cristina. Deconstrucción. Entrada del Diccionario de Hermenéutica por A. Ortiz-Osés y P. Lanzeros (directores). Bilbao: Universidad de Deusto, 1998.

SAMUEL, Raphael (ed.) People's History and Socialist Theory. London: History Workshop Journal, 1981.

VARELA R., Margarita; ÁVILA A., M. R., y IMELDA F., Teresa. La memoria. Definición, función y juego para la enseñanza de la medicina. Madrid: Editorial Médica Panamericana, 2005.

VIDAL, Fernando. Piaget antes de ser Piaget. Madrid: Ediciones Morata, 1998. 